\title{
Mimicking the Host Regulation of Salicylic Acid: A Virulence Strategy by the Clubroot Pathogen Plasmodiophora brassicae
}

\author{
Mohammad Djavaheri, ${ }^{1}$ Lisong Ma, ${ }^{1}$ Daniel F. Klessig, ${ }^{2}$ Axel Mithöfer, ${ }^{3}$ Gordon Gropp, ${ }^{1}$ and \\ Hossein Borhan ${ }^{1, \dagger}$ \\ ${ }^{1}$ Agriculture and Agri-Food Canada, Saskatoon Research Centre, 107 Science Place, Saskatoon, SK., S7N 0X2, Canada; \\ ${ }^{2}$ Boyce Thompson Institute, Cornell University, 533 Tower Road, Ithaca, NY 14853, U.S.A.; and ${ }^{3}$ Max-Planck-Institute for \\ Chemical Ecology, Beutenberg Campus, Hans-Knöll-Str. 8, D-07745 Jena, Germany
}

Accepted 23 August 2018.

The plant hormone salicylic acid (SA) plays a critical role in defense against biotrophic pathogens such as Plasmodiophora brassicae, which is an obligate pathogen of crucifer species and the causal agent of clubroot disease of canola (Brassica napus). $P$. brassicae encodes a protein, predicted to be secreted, with very limited homology to benzoic acid (BA)/SA-methyltransferase, designated PbBSMT. PbBSMT has a SA- and an indole-3-acetic acid-binding domain, which are also present in Arabidopsis thaliana BSMT1 (AtBSMT1) and, like AtBSMT1, has been shown to methylate BA and SA. In support of the hypothesis that $P$. brassicae uses PbBSMT to overcome SA-mediated defenses by converting SA into inactive methyl salicylate (MeSA), here, we show that PbBSMT suppresses local defense and provide evidence that PbBSMT is much more effective than AtBSMT1 at suppressing the levels of SA and its associated effects. Basal SA levels in Arabidopsis plants that constitutively overexpress PbBSMT compared with those in Arabidopsis wild-type Col0 (WT) were reduced approximately $80 \%$ versus only a $50 \%$ reduction in plants overexpressing AtBSMT1. PbBSMT-overexpressing plants were more susceptible to $P$. brassicae than WT plants; they also were partially compromised in nonhost resistance to Albugo candida. In contrast, AtBSMT1-overexpressing plants were not more susceptible than WT to either P. brassicae or A. candida. Furthermore, transgenic Arabidopsis and tobacco plants overexpressing PbBSMT exhibited increased susceptibility to virulent Pseudomonas syringae pv. tomato DC3000 (DC3000) and virulent Pseudomonas syringae pv. tabaci, respectively. Gene-mediated resistance to DC3000/AvrRpt2 and tobacco mosaic virus (TMV) was also compromised in Arabidopsis and Nicotiana tabacum 'Xanthi-nc' plants overexpressing PbBSMT, respectively. Transient expression of PbBSMT or AtBSMT1 in lower leaves of $N$. tabacum Xanthi-nc resulted in systemic acquired resistance (SAR)-like enhanced resistance to TMV in the distal systemic leaves. Chimeric grafting

${ }^{\dagger}$ Corresponding author: Hossein Borhan:

E-mail: Hossein.Borhan@agr.gc.ca

Funding: This research was supported by the Canadian Agri-Science Clusters Initiative AIP-P032 to H. Borhan and, in part, by US National Science Foundation grants IOS-0525360 and IOS-0820405 to D. F. Klessig.

*The $\boldsymbol{e}$-Xtra logo stands for "electronic extra" and indicates that five supplementary figures are published online.

๑) 2019 The American Phytopathological Society experiments revealed that, similar to $S A R$, the development of a PbBSMT-mediated SAR-like phenotype was also dependent on the MeSA esterase activity of NtSABP2 in the systemic leaves. Collectively, these results strongly suggest that PbBSMT is a novel effector, which is secreted by $P$. brassicae into its host plant to deplete pathogen-induced SA accumulation.

Clubroot disease of brassica species is caused by the biotrophic protist pathogen Plasmodiophora brassicae. Infection of canola (Brassica napus) by P. brassicae induces tumor and gall formation on the roots, which alters source/sink relationships, impedes water transport, and results in significant yield loss in severely infected crops (Dixon 2009). Plant pathogens including bacteria, true fungi, and protists, like oomycetes, secrete effectors to suppress plant defense responses through secretion of effectors into the host plant. Effectors are defined as small, secreted proteins produced by the pathogen to support pathogen colonization through the suppression of host immunity (Win et al. 2012). Several pathotypes of $P$. brassicae cause disease in their host species. The most prevalent pathotypes in Canada are pathotype $3(\mathrm{~Pb} 3)$, which is virulent on canola and pathotype $6(\mathrm{~Pb} 6)$, which mainly infects vegetable brassicas (Strelkov and Hwang 2014). Pb3 encodes approximately 590 secreted proteins, of which over 200 are small secreted proteins with plausible roles in pathogenicity (Rolfe et al. 2016).

Formation of galls by plant pathogens such as P. brassicae is a consequence of pathogen-triggered hormonal changes in the host. Auxin and cytokinin are two major hormones involved in the infection and development of root galls. Cytokinin levels are believed to rise in early stages of infection, while an increase in auxin levels occurs at later stages of infection. Genes that are potentially involved in cytokinin biosynthesis have been identified in P. brassicae, while the pathogen is believed to manipulate and utilize auxin produced by the host (Rolfe et al. 2016).

Based on the type of the pathogen encountered and the battery of specific effectors involved, plants employ a targeted array of deactivation or attenuation tactics against pathogens to restrict further ingress. To successfully defend against invading pathogens, plants have generally evolved two types of immunity responses, including pattern-triggered immunity (PTI) and effector-triggered immunity (ETI) (Jones and Dangl 2006). PTI is triggered by recognition of conserved microbial molecular patterns by associated plant pattern recognition receptors; while, in ETI, the recognition of a specific pathogen effector by 
its cognate plant resistance $(\mathrm{R})$ protein triggers a strong immune response, typically comprising a hypersensitive response.

Salicylic acid (SA) is the major plant hormone in defense against biotrophic pathogens, including clubroot (Lemarié et al. 2015; Ludwig-Müller et al. 2015; Rolfe et al. 2016; Vlot et al. 2009). Agarwal et al. (2011) showed that application of exogenous SA suppresses clubroot disease in Arabidopsis thaliana. To counteract SA-related defenses, it has been hypothesized that $P$. brassicae inactivates the plant-derived SA by converting it into biologically inactive methyl salicylate (MeSA) using a benzoic acid (BA)/SA methyltransferase protein (PbBSMT; National Center for Biotechnology Information accession number AFK13134) (Ludwig-Müller et al. 2015). PbBSMT identified in Pb3 (PbPT3Sc00026_A_1.308_1) encodes a predicted secreted protein. Sequence analysis revealed that PbBSMT has little overall homology to plant BSMT. However, PbBSMT shares partial homology to $S$-adenosyl methionine (SAM)-binding motifs of AtBSMT1 and NtSAMT1 (Ludwig-Müller et al. 2015; Rolfe et al. 2016). Moreover, recombinant PbBSMT made in Eschierchia coli has been shown to methylate SA and BA as well as anthranilic acid (Ludwig-Müller et al. 2015). Expression of PbBSMT was found to be very low in Brassica napus-infected roots during the first week after inoculation, then increased in week 2 , and peaked in weeks 3 and 4 (Rolfe et al. 2016). Upregulation of PbBSMT coincides with increased SA production in $P$. brassicaeinfected plants (Ludwig-Müller et al. 2015; Rolfe et al. 2016), suggesting a potential role for this enzyme in suppression of plant SA-dependent defense responses in Arabidopsis.

Many different plant species produce MeSA (Effmert et al. 2005). Volatile MeSA can function as fragrance for attraction of beneficial insects, like pollinators, to the plant (Goff and Klee 2006; Widhalm and Dudareva 2015). In addition to attraction, volatile MeSA is known to function as an airborne defense signal for plant-plant communication and tritrophic interactions of plant, herbivory insects, and parasitoids (Baldwin et al. 2006; Dempsey and Klessig 2012; Shulaev et al. 1997). MeSA was the first of several phloem-mobile signals for induction of systemic acquired resistance (SAR) to be identified in tobacco and Arabidopsis (Dempsey and Klessig 2012; Park et al. 2007; Vlot et al. 2008). Notably, MeSA has been reported to be transported from $P$. brassicae-infected roots to the aerial tissue (Ludwig-Müller et al. 2015).

A model has been proposed for SAR activation in Arabidopsis and tobacco by MeSA (Dempsey and Klessig 2012; Kumar and Klessig 2003; Liu et al. 2010; Park et al. 2007; Vlot et al. 2008). Rising SA in the primary infected leaves is converted to MeSA by NtSAMT1/AtBSMT1. MeSA accumulation is further enhanced in this tissue by SA inhibition of the MeSA esterase activity of tobacco SA-binding protein 2 (NtSABP2) or of the orthologous Arabidopsis MeSA esterases (AtMSEs). Following translocation to the distal systemic tissues, MeSA is converted back to SA by NtSABP2 (AtMSEs); the released SA then activates or primes systemic immune responses. A similar signaling pathway appears to operate in potato, since suppressing the activity of StMES1 compromised SAR (Manosalva et al. 2010). The levels of released SA in the systemic tissue are too low to inhibit the MeSA esterases, thereby allowing SA levels to increase.

Suppression of clubroot with exogenous SA (Agarwal et al. 2011) and the ability of recombinant PbBSMT to convert biologically active SA into MeSA, which is unable to induce immune responses (Ludwig-Müller et al. 2015), strongly suggests that PbBSMT is an effector. The most direct and rigorous test of this would be to delete or inactivate the PbBSMT and assess the effect on $P$. brassicae virulence. Since $P$. brassicae is not amenable to such genetic manipulation to help address this question, we determined the effects of overexpression of PbBSMT in transgenic Arabidopsis and tobacco. To assess why PbBSMT might function as an effector when it and the endogenous AtBSMT1 both can convert SA to MeSA, its effects were compared with those of overexpression of AtBSMT1. Here, we report that PbBSMT was much more effective than AtBSMT1 at both reducing endogenous and exogenous SA levels and at suppressing multiple levels of resistance, including nonhost and basal resistance as well as PTI and ETI.

\section{RESULTS}

PbBSMT is conserved among $P$. brassicae pathotypes and has a functional secretory signal peptide (SP).

The $P$. brassicae genome contains PbBSMT, with similarity to plant SAM-dependent carboxyl methyltransferase (methyltransferase-7 superfamily) (Rolfe et al. 2016). The well-studied BSMT gene in Arabidopsis (AtBSMT1, At3g11480) also belongs to the methyltransferase-7 superfamily (Liu et al. 2010, 2011). The $\mathrm{Pb} 3$ PbBSMT open reading frame is $1,134 \mathrm{bp}$ long, which encodes a 377-amino acid protein with very limited homology to AtBSMT1 (Ludwig-Müller et al. 2015). Importantly, recombinant PbBSMT can methylate SA and BA (Ludwig-Müller et al. 2015). PbBSMT is conserved in all the $P$. brassicae pathotypes sequenced to date (Rolfe et al. 2016). Despite differences in their host range (Rolfe et al. 2016), $P b B S M T$ alleles from $\mathrm{Pb} 3$ and $\mathrm{Pb} 6$ are $97 \%$ identical at the DNA level, while PbBSMT alleles from $\mathrm{Pb} 2, \mathrm{~Pb} 3, \mathrm{~Pb} 5$, and $\mathrm{Pb} 8$, and the European isolate e3 show $100 \%$ identity to $\mathrm{Pb} 3$ (not shown). PbBSMT has the signatures of plant pathogen effectors, which are the presence of an N-terminal SP and the lack of transmembrane and other subcellular targeting domains. To validate the function of PbBSMT predicted SP (PbBSMT-SP), we used a genetic assay in yeast, as described by Oh et al. (2009). The PbBSMT-SP was fused to the mature yeast invertase sequence, was cloned in the pSUC2 vector, and was transferred to the invertase-deficient mutant yeast strain YTK12. Transgenic strain YTK12 harboring the PbBSMT-SP fused to invertase was able to grow on a medium in which sucrose was replaced with raffinose, thereby demonstrating that PbBSMT-SP is a functional SP (Supplementary Fig. S1).

\section{Expression of 35S::PbBSMT in planta reduced SA levels and increased susceptibility to clubroot.}

Endogenous PbBSMT expression in P. brassicae increased soon after infection in canola root (Fig. 1A). Expression rose during formation of primary and secondary plasmodia and was highest during gall formation. To help assess the significance of PbBSMT activity for clubroot development, the transgenic Arabidopsis lines ectopically expressing AtBSMT1 (AtT) and PbBSMT (PbT) together with the wild type Col-0 (WT) control were evaluated for the effects of these methyltransferases on the levels of free and conjugated SA in leaves and roots. PbT plants were significantly more efficient than AtT plants at reducing the basal levels of SA and conjugated SA (Fig. 2A). Basal levels of biologically active free SA were reduced $80 \%$ in roots and leaves of PbT plants compared with those in WT, while the corresponding reductions in AtT were only approximately $50 \%$. Similarly, basal levels of conjugated SA were much more reduced in PbT than in AtT compared with those in WT, for both roots and leaves. Furthermore, MeSA was only detectable in the roots of PbT plants (32 $\mathrm{ng} \mathrm{g}^{-1}$ fresh weight). In agreement with stronger metabolism of endogenous SA in PbT leaves than in AtT leaves, the residual SA $18 \mathrm{~h}$ after infiltration of $0.25 \mathrm{mM}$ SA in PbT was only $2 \%$ of that remaining in infiltrated WT. In contrast, this level was reduced just $50 \%$ in AtT compared with WT. Thus, PbBSMT is much more effective than AtBSMT1 at 
metabolizing both endogenous and exogenous SA in roots and leaves of Arabidopsis.

Next we determined the effects of expressing $35 S:: P b B S M T$ and $35 S:: A t B S M T 1$ and the resulting enhanced metabolism of $\mathrm{SA}$ on clubroot induction by $\mathrm{Pb} 3$. The average number of infected cells containing secondary zoospores were over two times higher in the roots of PbT plants compared with those in AtT and WT plants. Furthermore, larger galls formed on PbT plants than on AtT and WT plants, which exhibited similar levels of infection (Fig. 1B and C). Additionally, an AtBSMT1 T-DNA knockout (AtBSMT1 KO, SALK 140496) was transformed by AtBSMT1 (Ask6) and PbBSMT (PsK5) and their susceptibility to clubroot was assessed. The infection rate was similar in the AtBSMT1 KO and AsK6 and comparable to that in WT. In contrast, PsK5 plants produced significantly larger galls than produced by the AtBSMT1 KO (Supplementary Fig. S5).

\section{Expression of $35 \mathrm{~S}:: \mathrm{PbBSMT}$ impaired nonhost resistance to Albugo candida.}

We have previously reported that Arabidopsis is generally not a host for Albugo candida isolate Ac2 V, which was isolated from Brassica spp. Interestingly, Ac2V is recognized in Arabidopsis Col-0 by the toll interleukin 1 receptor-nucleotide binding -leucine-rich repeat-type resistance gene WRR4 and the resulting resistance is SA dependent (Borhan et al. 2010). To test the effect of PbBSMT on SA-dependent defense, seedlings of $\mathrm{PbT}$ plants were inoculated with Ac2V. Trypan blue staining of infected cotyledons showed that ectopic overexpression of $35 S:: P b B S M T$ enabled hyphae branching and growth in $\mathrm{PbT}$ cotyledons compared with the immediate arrest of the pathogen at the site of infection in WT Col-0 (Fig. 2B). Impaired resistance was further evidenced by limited mycelial growth and sporulation on $\mathrm{PbT}$, which also occurred on Arabidopsis mutant eds 1 , which is defective in SA signaling, and on transgenic $N a h G$, which expresses a bacterial-derived SA hydroxylase that converts SA into inactive catechol (Fig. 2B). These results are consistent with PbBSMT suppressing SAmediated defenses. In contrast to $\mathrm{PbT}$, resistance in AtT to Ac2 $\mathrm{V}$ was similar to that in WT. Additionally, Ac2V showed partial mycelial growth in PsK5 that was similar to that in PbT (Fig. 2B), however its growth was strongly stopped in the ATBSMT1 KO and Ask6 cotyledons resembling nonhost resistance in WT Col-0.

PTI and basal resistance were compromised in plants expressing 35S::PbBSMT.

To evaluate whether PbBSMT alters PTI, PbT, and AtT, plants were inoculated with virulent Pseudomonas syringae pv. tomato DC3000 (DC3000) (Fig. 3). The SA signalingdefective $N a h G$ and $e d s l$ lines were again included as controls. Bacterial growth in PbT was compared with that in WT, AtT, $N a h G$, and $e d s 1$ at different times after inoculation. At day 3 postinoculation, DC3000 levels in PbT, AtT, NahG, and edsl were higher than that in WT (Fig. 3A), with $\mathrm{PbT}$ and $N a h G$ supporting the highest multiplication of DC3000, which was approximately 25 -fold more than that in WT. Similarly, increased bacterial growth was observed for Ask6 and PsK5

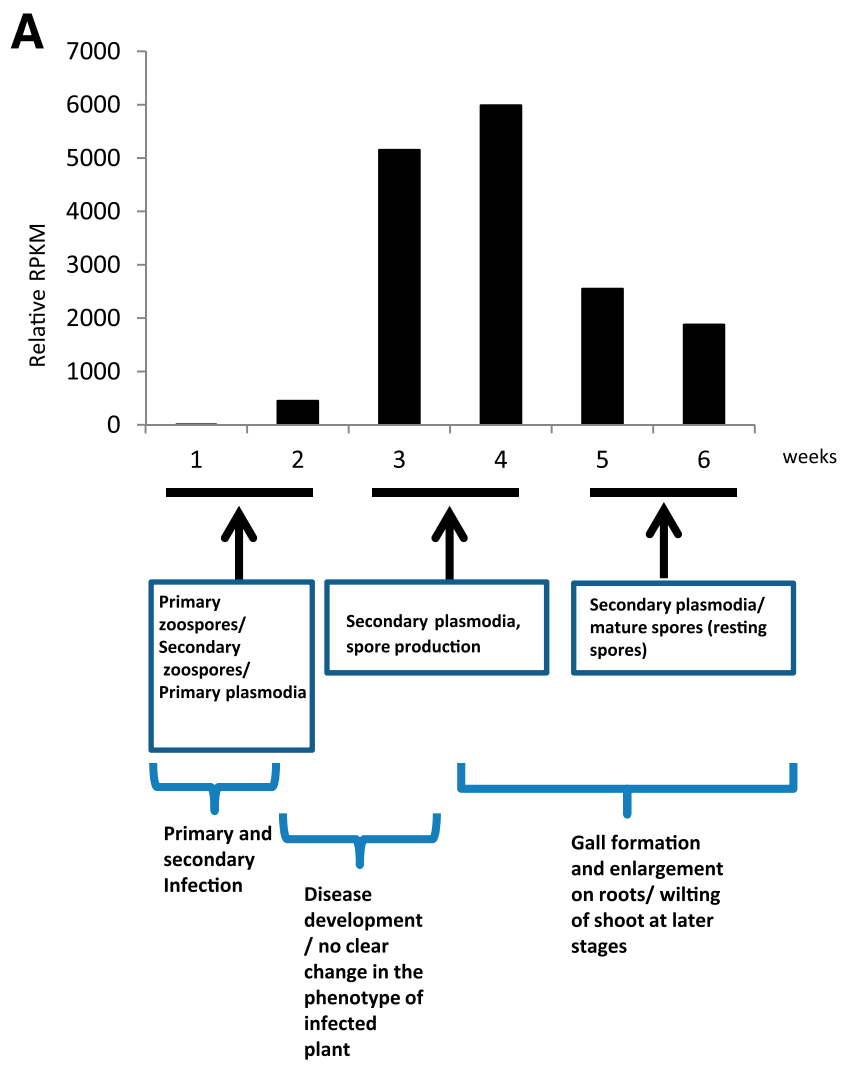

B



C

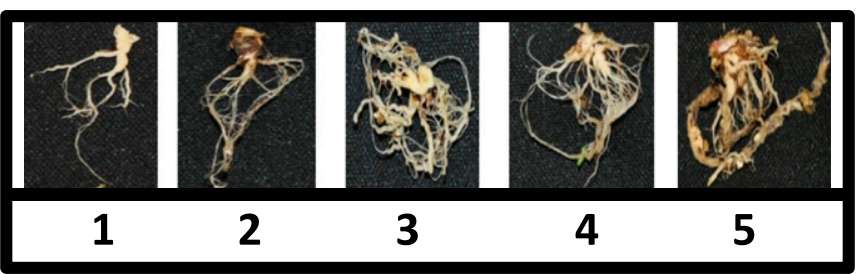

Fig. 1. Rating of clubroot disease on the roots of Arabidopsis plants inoculated with $10^{7}$ spores per milliliter of Plasmodiophora brassicae pathotype 3 (Pb3). A, Expression level of the P. brassicae benzoic acid/salicylic acid methyltransferase gene (PbBSMT) in canola (Brassica napus) roots infected with Pb3, at different growth stages of the pathogen. This panel is reproduced from data reported by Rolfe et al. (2016) to present a visual correlation between PbBSMT expression with pathogen growth and disease development in canola. B, Disease severity defined on scales of 0 to 5 , in which 0 was healthy roots with no galls and 5 was roots with severe gall formation. The percentage of roots in each group from a total of $100 \%$ is presented. Asterisks indicate statistically significant different distributions of disease severity classes between $35 S: \because P b B S M T$ expressing plants versus $35 S:: A t B S M T 1$ overexpressing line and WT (Chi-square test, $\alpha=0.05$ ). Three independent experiments were conducted with similar results. C, Images of plant roots infected with Pb3 representative of each step (1 to 5 ) of the scale. $\mathrm{RPKM}=$ reads per kilobase million. 
plants compared with that in the AtBSMT1 KO on day 3 after challenge-inoculation by virulent DC 3000 .

The expression of the SA-responsive marker gene $P R I$ was dramatically reduced in the leaves of $\mathrm{PbT}$ and $N a h G$ compared with WT, while eds 1 exhibited marginal reduction (Fig. 3B). Likewise, $P R I$ expression was significantly reduced in the infected leaves of PSK5 compared with the AtBSMT1 KO and Ask6 plants.

Next, the effect of $35 S:: P b B S M T$ overexpression on basal resistance was assessed following Pseudomonas syringae pv. tabaci 11584 infection of transgenic Nicotiana benthamiana (Nb-PbBSMT). Transgenic lines designated benth 7-6 and benth 7-8 supported a greater than sevenfold more growth of $P$. syringae pv. tabaci than WT $N$. benthamiana plants (Fig. 3C). Infiltration of $P$. syringae pv. tabaci into $N$. benthamiana leaves causes water-soaking and necrosis. Nb-PbBSMT plants infiltrated with $P$. syringae pv. tabaci developed necrotic symptoms almost 2 days earlier than WT plants. Moreover, lesion diameter was two to three times larger in transgenic $35 S:: P b B S M T$ plants. These results argue that in planta expression of $35 S:: P b B S M T$ impaired PTI induced by DC3000 in
Arabidopsis and basal resistance to $P$. syringae pv. tabaci in N. benthamiana.

\section{ETI was attenuated in Arabidopsis and tobacco plants expressing 35S::PbBSMT.}

To evaluate whether PbBSMT is able to alter ETI (also termed resistance $[R]$ gene-mediated resistance), $\mathrm{PbT}$ plants were inoculated with a strain of DC3000 harboring AvrRpt2, which is recognized by the Arabidopsis Col-0 $R$ gene RPS 2 . $\mathrm{PbT}$ plants were less resistant to DC3000/AvrRpt2 compared with the WT control, with bacterial levels 10-fold higher in $\mathrm{PbT}$ (Fig. 3D). In contrast and in agreement with Liu et al. (2010), bacterial levels in AtT were similar to those in WT.

To further assess effect of PbBSMT on ETI, two independent transgenic $N$. tabacum cv. Xanthi-nc (NN genotype) lines harboring 35S::PbBSMT (Nt-PbBSMT), designated Nt33-3 and $\mathrm{Nt} 33-4$, were tested for resistance to tobacco mosaic virus (TMV). N. tabacum Xanthi-nc contains the $R$ gene $N$, which confers ETI to TMV, including restriction of viral replication and development of necrotic lesions, referred to as a hypersensitive response (Deom et al. 1991). Nt33-3 and Nt33-4 were
A

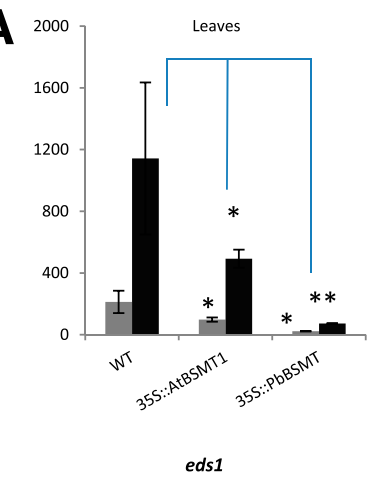

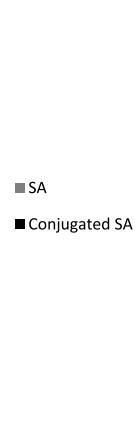

NahG

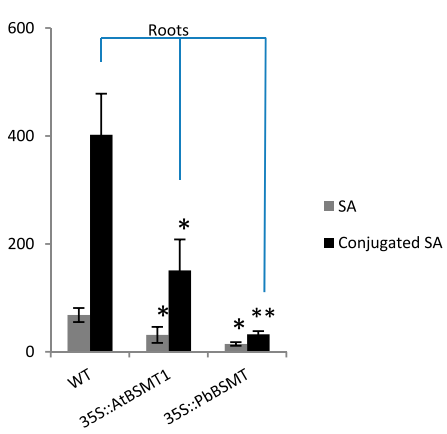

35S::PbBSMT (PbT)
35S::AtBSMT1 (AtT)

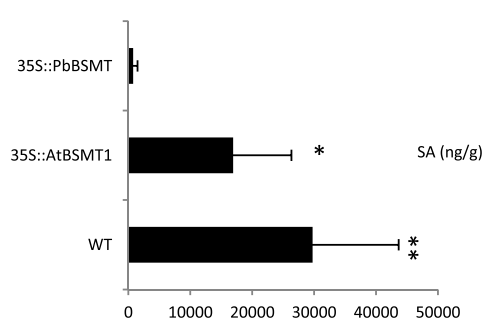

WT

B



Fig. 2. Levels of salicylic acid (SA) and its conjugated forms in Arabidopsis thaliana leaves and roots of noninoculated healthy plants constitutively expressing $35 S:: A t B S M T 1$ or $35 S:: P b B S M T$ compared with wild type (WT). A, Left and middle graphs show the levels of free and conjugated SA in the leaves and the roots, respectively, of healthy noninoculated plants; the right graph shows free SA in treated leaves $18 \mathrm{~h}$ after infiltration with $0.25 \mathrm{mM}$ SA. Values are the mean \pm standard deviation fresh weight measured in nanograms per gram. B, Nonhost response to Albugo candida race Ac2V in cotyledons of WT, transgenic 35S:: PbBSMT (PbT), transgenic 35S::AtBSMT (AtT), SA-deficient transgenic NahG, and SA signaling-defective eds1 mutant SG8-19. While Ac2V is nonvirulent on WT, its growth was facilitated in 35S::PbBSMT plants. In the lower panel, the extent of mycelial growth was visualized by trypan blue staining. The stained infected leaves correspond to the same plants shown in the middle and top panel. White dots on the lower surface of infected cotyledons show formation of pustules due to mycelial growth and sporulation. White scale bars are equal to $500 \mu \mathrm{m}$. Asterisks in A indicate statistically significant differences (one asterisk $[*$ ] indicates $P \leq 0.05$, two [**] $P \leq 0.01$, Student $t$ test) between the levels of SA or its conjugated form in 35S::PbBSMT and 35S::AtBSMT1 versus WT Col-0. For the left and middle graphs, statistical analysis was done between free SA (gray bar) and conjugated SA (black bar) separately. Two independent experiments were done for B with similar results. 
less resistant to TMV, as indicated by necrotic lesions that were five to eight times larger than those on the WT control (Fig. $3 \mathrm{E})$. Together, these two sets of data strongly argue that PbBSMT suppresses ETI.

\section{Local 35S::PbBSMT expression} in tobacco-induced SAR to TMV.

PbBSMT methylates SA to form MeSA (Ludwig-Müller et al. 2015), similar to its tobacco (NtSAMT1) and Arabidopsis (AtBSMT1) orthologs. NtSAMT1 is involved at the onset of SAR in the primary leaves in which this SAR signal is generated (Liu et al. 2011; Park et al. 2007; Vlot et al. 2008). To examine whether PbBSMT or AtBSMT1 could induce SARlike phenotype, $35 S:: P b B S M T$ or $35 S:: A t B S M T 1$ were transiently expressed in three lower leaves of $N$. tabacum Xanthi-nc and, 1 week later, the upper systemic leaves were challenged with TMV. PbBSMT was detected in the infiltrated leaves of $N$. tabacum Xanthi-nc as early as 4 days postinoculation (dpi) and persisted up to 16 dpi (data not shown). Average TMV lesion size was reduced by 70 and $50 \%$ in the upper systemic leaves of plants transiently expressing $35 S:$ : $P b B S M T$ or $35 S:: A t B S M T 1$, respectively, in the lower leaves compared with that on plants not expressing either, i.e., mockinfiltrated (Fig. 4). These results revealed that expression of either pathogen or plant BSMT was enough to induce systemic resistance to TMV in Xanthi-nc resembling the pathogeninduced SAR.

\section{NtSABP2 was required}

for enhanced resistance induced by PbBSMT.

Since conversion of inactive MeSA back into active SA by NtSABP2 in the systemic tissues is essential for SAR (Park et al. 2007), we tested whether NtSABP2 is required for PbBSMT-induced SAR-like resistance, using $N$. tabacum Xanthi-nc plants in which SABP2 was silenced (siSABP2). $35 S:: P b B S M T$ was transiently expressed in the lower leaves of siSABP2 and WT plants, which was followed a week later with TMV inoculation of the upper systemic leaves. Again, similar experiments were conducted using transient expression of $35 S:: A t B S M T 1$ as a positive control. PbBSMT- as well as
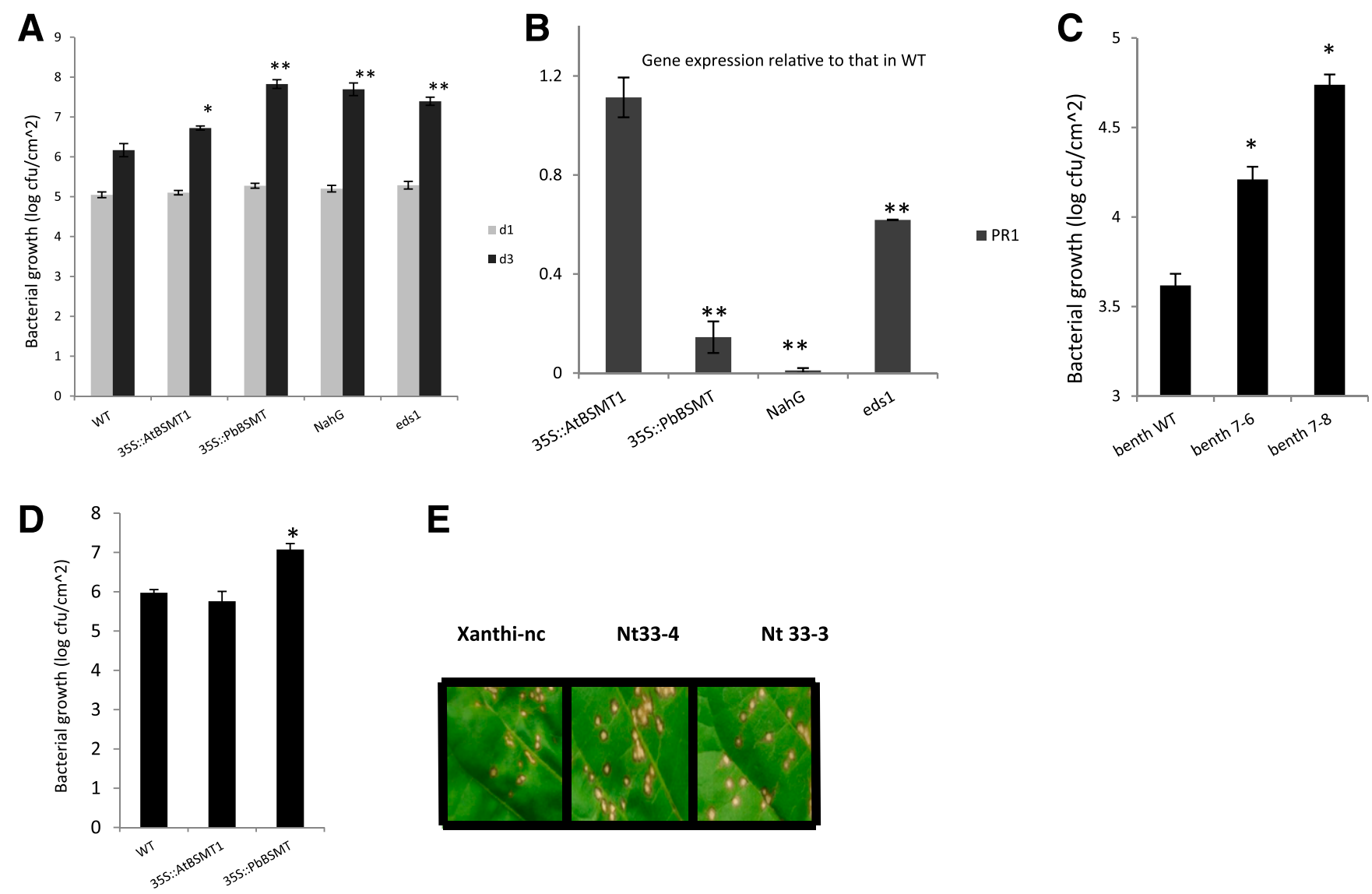

$\mathbf{E}$

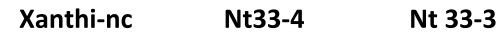

Fig. 3. Pattern- and effector-triggered immunity (PTI and ETI, respectively) in Arabidopsis and tobacco plant expressing $35 S:: P b B S M T$ and $35 S:: A t B S M T 1$. For PTI response, three to four leaves of five Arabidopsis thaliana and Nicotiana benthamiana plants were infiltrated with virulent Pseudomonas syringae pv. tomato DC3000 (DC3000) or Pseudomonas syringae pv. tabaci 11584, respectively. ETI was examined by inoculating three leaves of five individual A. thaliana or N. tabacum 'Xanthi-nc' with DC3000/AvrRPT2 or tobacco mosaic virus (TMV), respectively. A, Wild type (WT), transgenic 35S::PbBSMT (PbT), transgenic 35S::AtBSMT (AtT), salicylic acid (SA)-deficient transgenic NahG, and SA signaling-defective $e d s 1$ mutant SG8-19 were infiltrated with $10^{6}$ CFU $\mathrm{ml}^{-1}$ of DC3000; at 1 and 3 days postinoculation, the average number of colony-forming units in the infiltrated leaves was calculated and are presented on a log to the base 10 scale. B, mRNA levels of SA-responsive marker gene PR1 at day 3 postinoculation with DC3000 were quantified with quantitative real-time polymerase chain reaction and are presented relative to those in WT. C, The average number of colony-forming units of Pseudomonas syringae pv. tabaci 115843 days postinoculation of WT $N$. benthamiana and of two independent $35 S:: P b B S M T$-expressing transgenic lines, benth7-6 and benth7-8, are presented on a $\log$ scale. D, The average number of colony-forming units of DC3000/AvrRPT2 in 35S::PbBSMT-expressing Arabidopsis compared with WT and 35S:: AtBSMT1 plants at 3 days postinoculation are presented on a log scale. E, TMV lesions on WT $N$. tabacum Xanthi-nc and on two independent $35 S:: P b B S M T$ expressing $N$. tabacum Xanthi-nc transgenic lines, Nt33-3 and Nt33-4. In A (bacterial growth on day 3) and D, asterisks indicate significant difference (one asterisk [*] indicates $P \leq 0.05$, two [**] $P \leq 0.01$, Student $t$ test) between the average values for each plant versus WT Col-0. In B, asterisks indicate significant difference (one asterisk [*] indicates $P \leq 0.05$, two [**] $P \leq 0.01$, Student $t$ test) between the average relative values for each plant to that in WT versus WT Col0 as control. In $\mathrm{C}$, asterisks represent statistical differences between (one asterisk [*] indicates $P \leq 0.05$, Student $t$ test) each transformant versus WT $N$. benthamiana. For each experiment, at least two independent experiments were conducted with similar results. 
AtBSMT1-induced systemic resistance against TMV was abolished in siSABP2, indicating that SABP2 is required for their induction of SAR-like phenotype (Fig. 4).

The role of NtSABP2 in PbBSMT-mediated SAR-like induction was further investigated with chimeric grafts between WT N. tabacum Xanthi-nc and siSABP2. 35S::PbBSMT was infiltrated into three lower leaves of the rootstock and, 1 week later, TMV was inoculated on the systemic leaves of the scion (Fig. 5A). At 4 to 7 dpi, lesion size was compared with those on

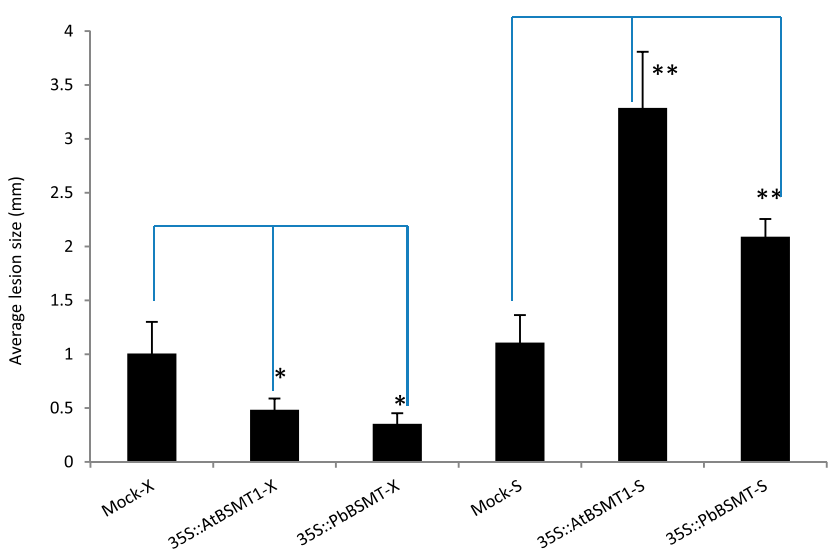

Fig. 4. $P b B S M T$ and AtBSMT1 induced a systemic acquired resistance (SAR)-like phenotype in Nicotiana tabacum 'Xanthi-nc' against tobacco mosaic virus (TMV). Average TMV lesion size on the upper leaves of Xanthi-nc (X) or its salicylic acid (SA)-binding protein 2-silenced mutant NtSiSABP2 (S) when $35 S:: A t B S M T 1$ or $35 S: \because P b B S M T$ were transiently expressed in the three lower leaves compared with average lesion size on plants whose lower leaves were not expressing $35 S:: A t B S M T 1$ or $35 S:$ : $P b B S M T$. Mean \pm standard deviation values are presented. Statistical analysis on average induction levels in N. tabacum Xanthi-nc (X) and NtSiSABP2 (S) plants was done separately. Asterisks indicate statistically significant differences (one asterisk [*] indicates $P \leq 0.05$, two [**] $P \leq 0.01$, Student $t$ test). Two independent experiments were done with similar results. control plants in which the rootstock was mock infiltrated before TMV was inoculated on a scion. Again $35 S: \because P b B S M T$ infiltrated rootstocks suppressed TMV lesion size on systemic leaves compared with mock-infiltrated plants (Fig. 5B). Similarly, when siSABP2 was used as rootstock, PbBSMT induced SAR-like resistance in WT $N$. tabacum Xanthi-nc scions. However, the PbBSMT-induced SAR-like phenotype was disrupted when the siSABP2 scion was grafted onto the $35 S:$ : PbBSMT-expressing rootstock (Fig. 5B). These results together confirm that PbBSMT can induce systemic resistance, which is phenotypically similar to pathogen-induced SAR and this is dependent on the activity of NtSABP2 in the systemic tissues.

\section{PbBSMT suppresses SA-mediated retardation of root growth in Arabidopsis.}

To effectively defend themselves, plants infected by $P$. brassicae accumulate large quantities of SA in their roots (Lemarié et al. 2015). While SA accumulation in the plants serves to protect them from the invading pathogen, high levels of SA can impose fitness costs (van Hulten et al. 2006). To prevent the negative impact of high SA levels, plants convert this highly active phytohormone into inactive metabolites, including MeSA (Dempsey et al. 2011). To assess whether PbBSMT or AtBSMT1 could suppress the toxic effect of high SA levels on root growth WT, AtT, and PbT, plants were grown on Murashige Skoog (MS) agar without or with different concentrations of SA added to the media (Fig. 6A). While root growth was similarly retarded in WT and AtT by SA, PbT exhibited increase tolerance to SA. In addition, when 4-day-old seedlings growing on MS media without SA were transferred onto MS media containing SA, roots of PbT plants grew longer than those of either WT or AtT (Fig. 6B).

\section{Altered hormonal levels in $\mathrm{PbT}$ and AtT plants with reduced levels of $\mathrm{SA}$.}

Clubroot infection results in changes in the content of plant hormones, including SA, auxin, cytokinin, jasmonic acid (JA),
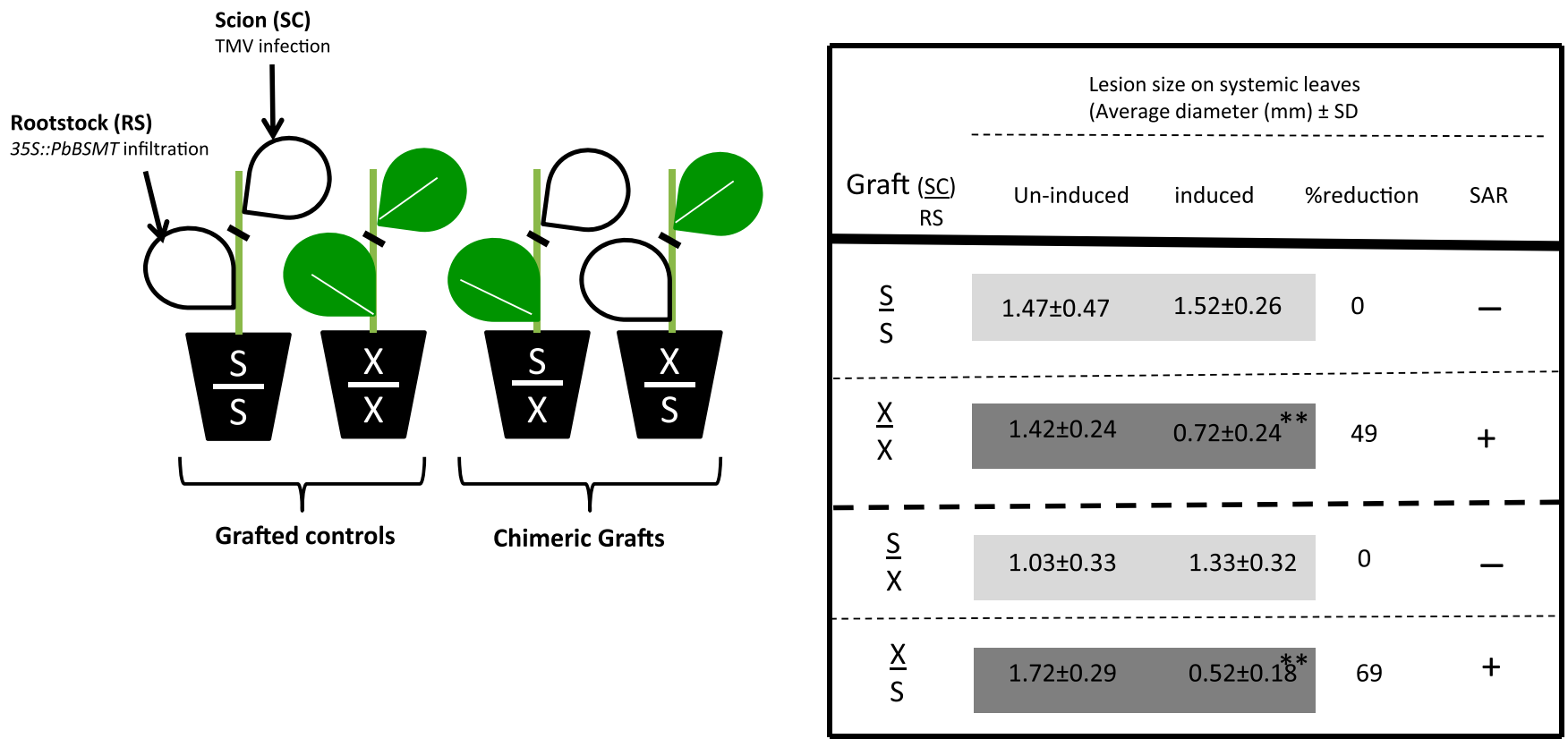

Fig. 5. PbBSMT and AtBSMT1 induced a systemic acquired resistance (SAR)-like phenotype in Nicotiana tabacum 'Xanthi-nc' against tobacco mosaic virus (TMV). Average TMV lesion size on the upper leaves of N. tabacum Xanthi-nc (X) or its salicylic acid (SA)-binding protein 2-silenced mutant NtSiSABP2 (S) when $35 S:: A t B S M T 1$ or $35 S:: P b B S M T$ were transiently expressed in the three lower leaves compared with average lesion size on plants whose lower leaves were not expressing $35 S:: A t B S M T 1$ or $35 S:: P b B S M T$. The mean \pm standard deviation values are presented. Statistical analysis on average induction levels in N. tabacum Xanthi-nc (X) and NtSiSABP2 (S) plants was done separately. Asterisks indicate statistically significant differences (one asterisk [*] indicates $P \leq 0.05$, two [**] $P \leq 0.01$, Student $t$ test). Two independent experiments were done with similar results. 
and abscisic acid (ABA) in infected roots (Jülke and LudwigMüller 2016; Lemarié et al. 2015). Moreover, the interplay among SA and other plant hormones, including JA, ethylene (ET), ABA, and indole-3-acetic acid (IAA), have been documented in plants responding to various environmental cues. In addition to reduced SA levels (Fig. 2A), PbT and AtT also had changes in other hormones. For example, levels of the ET precursor 1-aminocyclopropane-1-carboxylate (ACC) also was depressed in both leaves and roots of PbT and AtT (Supplementary Fig. S2), while levels of cytokinin and gibberellins were reduced only in the roots of PbT and AtT compared with those in WT (Supplementary Figs. S3 and S4). Levels of ABA and its metabolites were lower only in the leaves and roots of
AtT. Of particular interest were the levels of the auxins, since AtBSMT1 and PbBSMT have an apparent indole-3-acetate (IAA) carboxyl methyltransferase domain. The levels of the biologically active IAA and its conjugates $N$-(Indole-3-ylacetyl)-aspartic acid (IAA-Asp) and $N$-(Indole-3-yl-acetyl)glutamic acid (IAA-Glu) were not significantly different in $\mathrm{PbT}$, AtT, and WT.

\section{DISCUSSION}

Ludwig-Müller and coworkers (2015) proposed that $P$. brassicae overcomes SA-mediated defenses by secreting PbBSMT, which can convert SA into inactive MeSA. Together,

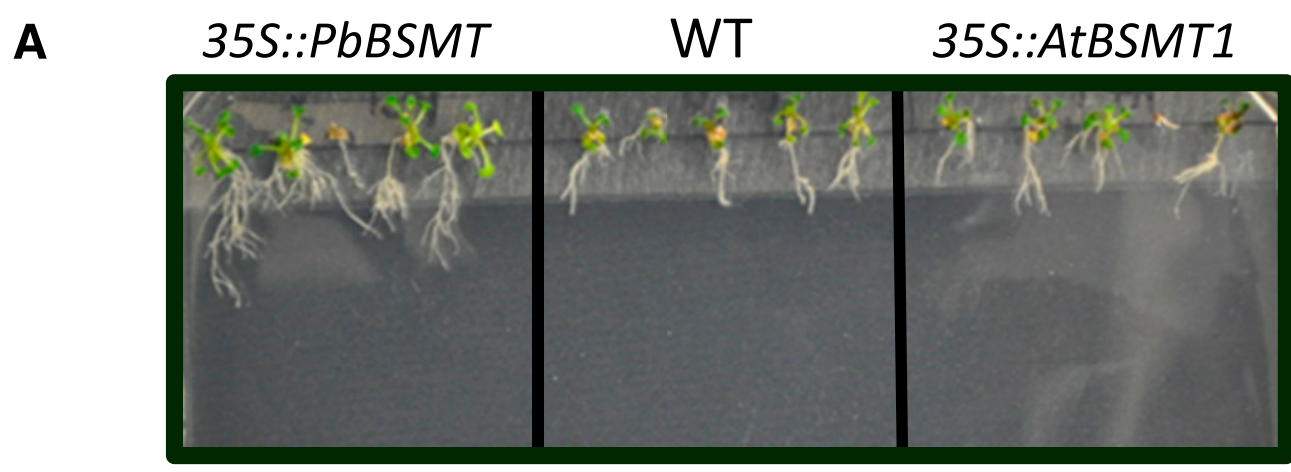

B


C
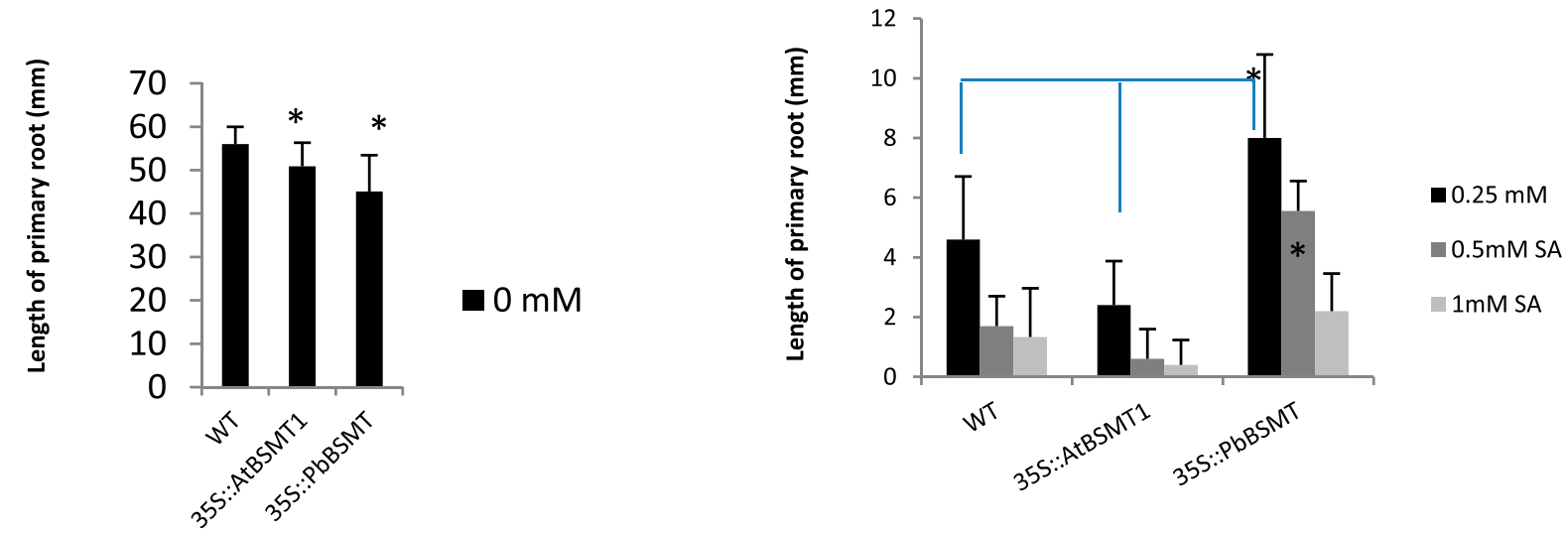

Fig. 6. Effect of salicylic acid (SA) on root growth of wild type (WT) and 35S::AtBSMT1- or 35S::PbBSMT-overexpressing Arabidopsis. A, Growth on Murashige Skoog (MS) agar containing $0.25 \mathrm{mM} \mathrm{SA}$ for 21 days. B, Average length of primary root at 21 days after planting when seeds were germinated directly on MS media containing various concentrations of SA. C, Average length of primary root when seeds were first grown for 4 days on MS agar without SA (left panel) before transfer of the seedlings to MS media containing various concentrations of SA; root length on day 21 minus the root length on day 4, when the seedling were transferred, was plotted (right panel). Asterisks indicate significant difference between each of the plants versus WT Col-0 (one asterisk [*] indicates $P \leq 0.05$, Student $t$ test). Two independent experiments were done with similar results. 
the results presented here strongly support that proposition. Moreover, they provide an explanation for why PbBSMT could suppress SA-mediated defenses while the endogenous BSMT does not. Ectopic overexpression of PbBSMT more effectively reduced levels of endogenous and exogenous SA and its conjugate form than overexpression of AtBSMT1. It also suppressed multiple levels of resistance from nonhost to ETI in Arabidopsis and tobacco, including resistance to $P$. brassicae. In contrast, overexpression of AtBSMT1 generally failed to reduce resistance including to $P$. brassicae. Our finding that overexpression of $B S M T$ reduced resistance to virulent bacteria is consistent with the results of Liu et al. (2010), who reported that overexpression of AtBSMT1 in Arabidopsis resulted in elevated MeSA levels, whereas levels of SA, its conjugate, and resistance to virulent Pseudomonas syringae were substantially reduced. The differential effects of $P$. brassicae-secreted BSMT versus endogenous BSMT likely reflects the higher level of the $P$. brassicae enzyme in the infected tissue than that of the endogenous enzyme, whose expression is not induced by $P$. brassicae infection (S. Rolfe, personal communication). It may also be due to possibly higher specific activity of the pathogen enzyme than the plant enzyme, which is suggested by the higher level of conversion of SA to MeSA in PbT plants than in AtT plants.

Similarly, the finding that $\mathrm{PbT}$ plants were more resistant to SA-mediated retardation of seed germination and root growth while AtT plants were as sensitive as WT to SA again suggests that the pathogen enzyme is more potent. Importantly, rising levels of SA in infected roots could suppress root growth. The ability of PbBSMT to reduce SA levels would facilitate the growth of the very tissue this biotroph needs to multiply. Thus, PbBSMT inactivation of SA not only suppresses SA-mediated defenses but also suppresses its toxic effect on root growth. It should be noted that, in our experiments, PbBMST protein was expressed ubiquitously while, in $P$. brassicae-infected plants, mature PbBSMT protein is likely concentrated within infected tissue. Effects of root-localized PbBSMT on the defense in distal tissues need to be studied.

Comparison of the effects of overexpression of PbBSMT to those caused by a mutation that alters SA signaling (eds1) or by a transgene affecting SA accumulation $(N a h G)$ provides additional support that PbBSMT functions to suppress SA-mediated defenses. Interestingly, although $\mathrm{PbT}$ and $\mathrm{NahG}$ both exhibited reduced resistance to Albugo candida and DC3000 while resistance to $\mathrm{Pb} 3$ was compromised in $\mathrm{PbT}$, it was not in NahG. This difference suggests that perhaps PbBSMT has an additional virulence activity besides inactivating SA. In agreement, Lovelock et al. (2016) showed reduced susceptibility in ColNahG plants infected by two isolates of $P$. brassicae.

Several plant hormones including IAA, cytokinins, JA, ET, brassinosteroids (BR), and ABA have been shown to have roles in clubroot disease development in crucifers, with SA, BR, and IAA having the most prominent roles (Ludwig-Müller 2014; Rolfe et al. 2016). Interconnection between many hormone pathways has been described as hormone cross-talk. This phenomenon is defined to act as a general plant strategy in various stress responses to environmental and internal cues (Vos et al. 2015). In addition to SA depletion (in the shoots and roots), PbBSMT overexpression resulted in reduction in basal levels of ACC (in the shoots and roots) and cytokinins in transgenic plants. While elevated cytokinin is thought to be important for gall development, $\mathrm{PbT}$ plants supported growth of larger galls than WT plants despite having lower basal cytokinin levels. Perhaps, in infected roots, cytokinin production is manipulated by the pathogen, since annotated genes of $\mathrm{Pb} 3$ include those with a potential role in cytokinin regulation (Rolfe et al. 2016).
In summary, our data argue that PbBSMT functions as a virulent factor by suppressing SA-mediated defenses against biotrophic pathogens through its conversion of biologically active SA into inactive MeSA. In addition, inactivation of SA suppresses its toxic effect on root growth, which also would facilitate $P$. brassicae colonization leading to clubroot disease.

\section{MATERIALS AND METHODS}

\section{Plant materials.}

Arabidopsis and tobacco plants were grown on a soilless potting mix and were kept in growth cabinets with $8 \mathrm{~h}$ of light $\left(100 \mu \mathrm{mol} / \mathrm{m}^{2} / \mathrm{s}\right)$ at 22 (light) and $20^{\circ} \mathrm{C}$ (dark) and $70 \%$ relative humidity. A. thaliana plants overexpressing Arabidopsis BSMT (AtBSMT1, AtT), Col-NahG, and Col-eds1 have been described elsewhere (Borhan et al. 2008; Liu et al. 2010; Van Wees and Glazebrook 2003). Nicotiana tabacum Xanthi-nc silenced for NtSABP2 was described by Park et al. (2007).

To compare the efficacy of AtBSMT1 and PbBSMT in detoxification of a high concentration of SA, seeds were surfacesterilized and were sown on MS agar medium supplemented with $0.5 \%$ sucrose. SA was added to MS agar plates at a concentration of $0.25,0.5$, and $1 \mathrm{mM}$. After 4 days of stratification at $4{ }^{\circ} \mathrm{C}$, the petri dishes were transferred and were positioned vertically under a short-day photoperiod $\left(8 \mathrm{~h}\right.$ of light at $\left.22^{\circ} \mathrm{C}\right)$. Additionally, seeds were grown on MS media for 4 days, and then, seedlings were transferred onto new MS plates containing various concentrations of SA. The average growth rate of plant roots was calculated.

Systemic expression of NtSABP2 was shown to be necessary for pathogen-induced SAR (Park et al. 2007). To test whether $N t S A B P 2$ expression in distal leaves is also important for induction of resistance against TMV by PbBSMT, grafting was done using $N$. tabacum Xanthi-nc. and $N$. tabacum siSABP2. After successful grafting, three lower leaves of rootstock were infiltrated by either $35 S: \because P b B S M T$ or $35 S:: G F P$ as mock. Later, three leaves on the scion were infected by TMV and disease was scored 4 to 7 days later.

\section{Pathogens and bioassays.}

Clubroot pathology on Arabidopsis plants was carried out as soil drench of an inoculum of the single spore (ss) isolate of Plasmodiophora brassicae pathotype 3 (provided by S. E. Strelkov, University of Alberta) prepared at a concentration of $2 \times 10^{7} \mathrm{ss} \mathrm{ml}^{-1}$. Four weeks after inoculation, 20 to 25 roots were analyzed for the appearance of root galls. Clubroot symptoms were scored from 0 to 5 for no gall to massive galls on the crown and entire root system. Disease severity was calculated as the average percentage of disease for each rating scale. Pseudomonas syringae pv. tomato DC3000 (starting inoculum $1 \times 10^{6} \mathrm{CFU} \mathrm{ml^{-1 }}$ ) and DC3000 harboring AvrRpt2 (starting inoculum $1 \times 10^{5} \mathrm{CFU} \mathrm{ml}^{-1}$ ) were used to study PTI (also called PAMP-triggered immunity) and ETI in 5-week-old Arabidopsis plants, respectively (Liu et al. 2010). Three leaves of five individual plants were infiltrated with a suspension of each bacterial strain and average numbers of bacteria were calculated on various days. Quantitative real-time PCR (PCR) analysis of AtPRl transcript was done on Arabidopsis leaves collected at 3 days after inoculation by DC3000 (SanchezVallet et al. 2010).

Pseudomonas syringae pv. tabaci 11584 (ATCC11528 provided by D. S. Guttman, University of Toronto) (P. syringae pv. tabaci) was used to infiltrate the leaves of 4-week-old Nicotiana benthamiana plants $\left(10^{5} \mathrm{CFU} \mathrm{ml}^{-1}\right)$. Average lesion diameter as well as average bacterial numbers on day 7 after inoculation were calculated. TMV (provided by ?? Kovalchuk, University of Lethbridge) and Albugo candida race Ac2V that 
were maintained on the host were used to inoculate $N$. tabacum Xanthi-nc and Arabidopsis as described before (Borhan et al. 2008; Park et al. 2007). Albugo mycelial growth was observed under the light microscope upon staining with trypan blue (van Damme et al. 2008). Average TMV lesion diameter was measured at 4 to 7 days after inoculation.

\section{Cloning, transformation, confocal laser scanning microscopy, and the yeast trap system.}

Gateway cloning vectors pDONR/zeo and pEarleyGate 100 were used to clone cDNA of AtBSMT1 (At3g11480) or PbBSMT (PbPT3Sc00026_A_1.308_1) and to transform Arabidopsis Col-0. Additionally, $N$. benthamiana and $N$. tabacum Xanthi-nc were also transformed with cDNA of PbBSMT. Droplet digital PCR (Whale et al. 2012) was used to determine the transgene copy number and select transformants with a single insertion. In Arabidopsis, the phosphinothricin (bar) selectable marker gene was amplified using the primer set 5'-GGGGCCCGCGTAG GCGATGC-3' (forward) and 5'-CGTACCGAGCCGCAGGAA CC-3' (reverse) and was detected with the 6-carboxyfluoresceinlabeled probe 5'-ATCCCTGGCTCGTCGCCGAGGTGG-3'. The Arabidopsis alcohol dehydrogenase (AtADH) reference gene was amplified using the primer set 5'-CTTCCACGTTCAGT GAGTACA-3' (forward) and 5'-CTCCTAACCCAGTAGACA AACC-3' (reverse) and was detected with the hexachlorofluorescein (HEX)-labeled probe 5'-AGCAACCTGACCAG AGTGAACCAC- $3^{\prime}$. Individual single copy lines were allowed to self-pollinate and independent homozygous lines were selected for further investigation. The expressions of PbBSMT in $N$. benthamiana and $N$. tabacum Xanthi-nc transgenic lines were also determined, using droplet digital PCR. The phosphinothricin (bar) selectable marker gene was amplified using the same primer set as described above. The $N b A D H$ reference gene was amplified using the primer set 5'-TCAAGTATCGATTCGTG CTC-3' (forward) and 5'-AGAGCTAAGCAAAGACAAA GAT-3' (reverse) and was detected with the HEX-labeled probe 5'-TTGGCAACCATTCCAACTCTGCTT-3'. The NtAllylADH reference gene was amplified using the primer set $5^{\prime}$-CACTG CTCTAGTTGGTCTCTTC-3' (forward) and 5'-GCGTAAACA CAAACAGCTCTAC-3' (reverse) and was detected with the HEX-labeled probe 5'-AAAGCAAGTCGTGATGGTTTCG CG-3'. Insert copy numbers were calculated in $N$. benthamiana and $N$. tabacum relative to the $N b A D H$ or NtAllylADH reference genes, respectively, which are present as one copy in the $N$. benthamiana and two in the $N$. tabacum genomes.

For the yeast signal sequence trap experiment (Oh et al. 2009), nucleotide sequences corresponding to positions 1 to 21 amino acids (SP fragment) of PbBMST was PCR-amplified. Yeast transformation was performed according to the protocol listed in the Yeastmaker Yeast Transformation System 2 (Clontech). The invertase-negative yeast strain YTK12 was transformed with a $p S U C 2:: S P(P b B S M T)$ construct or empty vector $p S U C 2$ (negative control). Transformed colonies were transferred to CMD-W (without Trp) plates and were incubated at $30^{\circ} \mathrm{C}$ for 3 days. For the invertase secretion assay, transformed colonies were replica-plated on CMD-W plates and YPRAA ( $1 \%$ yeast extract, $2 \%$ peptone, $2 \%$ raffinose, and $2 \mu \mathrm{g} / \mathrm{ml}$ antimicyn A), containing raffinose and lacking glucose. After 3 days incubation at $30^{\circ} \mathrm{C}$, the plates were checked for growth and were photographed.

\section{Hormonal assays.}

Plant hormones and their derivatives, including SA, auxin, cytokinin, gibberellic acid, ABA, and the precursor of ET (ACC), were quantified in the leaves and roots of Arabidopsis plants using Ultra-performance liquid chromatographicelectrospray ionization-tandem mass spectrometry in the
Aquatic and Crop Resource Development lab at the National Research Council Canada, Saskatoon and the Max Planck Institute for Chemical Ecology, Jena, Germany (Chao et al. 2016; Chauvaux et al. 1997; Kodja et al. 2015; Lulai et al. 2016; Lulsdorf et al. 2013; Murmu et al. 2014; Vadassery et al. 2012).

\section{ACKNOWLEDGMENT}

Authors thank following individuals for providing materials: D. S. Guttman, University of Toronto for $P$. syringae pv. tabaci $11584 ; \mathrm{X}$. Li, University of British Columbia for DC3000/AvrRpt2; and I. Kovalchuk, University of Lethbridge for tobacco mosaic virus. We thank K. Rozwadowsky, Agriculture and Agri-Food Canada, Saskatoon Research Centre for sharing Arabidopsis reference gene for assays, C. Coutu for help with confocal laser scanning microscopy, and M. Reichelt for support in phytohormone analysis.

\section{LITERATURE CITED}

Agarwal, A., Kaul, V., Faggian, R., Rookes, J. E., Ludwig-Müller, J., and Cahill, D. M. 2011. Analysis of global host gene expression during the primary phase of the Arabidopsis thaliana-Plasmodiophora brassicae interaction. Funct. Plant Biol. 38:462-478.

Baldwin, I. T., Halitschke, R., Paschold, A., von Dahl, C. C., and Preston, C. A. 2006. Volatile signaling in plant-plant interactions: "Talking trees" in the genomics era. Science 311:812-815.

Borhan, M. H., Gunn, N., Cooper, A., Gulden, S., Tör, M., Rimmer, S. R., and Holub, E. B. 2008. WRR4 encodes a TIR-NB-LRR protein that confers broad-spectrum white rust resistance in Arabidopsis thaliana to four physiological races of Albugo candida. Mol. Plant-Microbe Interact 21:757-768.

Borhan, M. H., Holub, E. B., Kindrachuk, C., Omidi, M., BozorgmaneshFrad, G., and Rimmer, S. R. 2010. WRR4, a broad-spectrum TIR-NBLRR gene from Arabidopsis thaliana that confers white rust resistance in transgenic oilseed brassica crops. Mol. Plant Pathol. 11:283-291.

Chao, W. S., Doğramaci, M., Horvath, D. P., Anderson, J. V., and Foley, M. E. 2016. Phytohormone balance and stress-related cellular responses are involved in the transition from bud to shoot growth in leafy spurge. BMC Plant Biol. 16:47.

Chauvaux, N., Van Dongen, W., Esmans, E. L., and Van Onckelen, H. A. 1997. Quantitative analysis of 1-aminocyclopropane-1-carboxylic acid by liquid chromatography coupled to electrospray tandem mass spectrometry. J. Chromatogr. 775:143-150.

Dempsey, D. A., and Klessig, D. F. 2012. SOS-Too many signals for systemic acquired resistance? Trends Plant Sci. 17:538-545.

Dempsey, D. A., Vlot, A. C., Wildermuth, M. C., and Klessig, D. F. 2011. Salicylic acid biosynthesis and metabolism. Arabidopsis Book 9:e0156.

Deom, C. M., Wolf, S., Holt, C. A., Lucas, W. J., and Beachy, R. N. 1991. Altered function of the tobacco mosaic virus movement protein in a hypersensitive host. Virology 180:251-256.

Dixon, G. R. 2009. The occurrence and economic impact of Plasmodiophora brassicae and clubroot disease. J. Plant Growth Regul. 28: 194-202.

Effmert, U., Saschenbrecker, S., Ross, J., Negre, F., Fraser, C. M., Noel, J. P., Dudareva, N., and Piechulla, B. 2005. Floral benzenoid carboxyl methyltransferases: From in vitro to in planta function. Phytochemistry 66:1211-1230.

Goff, S. A., and Klee, H. J. 2006. Plant volatile compounds: Sensory cues for health and nutritional value? Science 311:815-819.

Jones, J. D. G., and Dangl, J. L. 2006. The plant immune system. Nature 444:323-329.

Jülke, S., and Ludwig-Müller, J. 2016. Response of Arabidopsis thaliana roots with altered lipid transfer protein (LTP) gene expression to the clubroot disease and salt stress. Plants 5:2.

Kodja, H., Noirot, M., Khoyratty, S. S., Limbada, H., Verpoorte, R., and Palama, T. L. 2015. Biochemical characterization of embryogenic calli of Vanilla planifolia in response to two years of thidiazuron treatment. Plant Physiol. Biochem. 96:337-344.

Kumar, D., and Klessig, D. F. 2003. High-affinity salicylic acid-binding protein 2 is required for plant innate immunity and has salicylic acid-stimulated lipase activity. Proc. Natl. Acad. Sci. U.S.A. 100:16101-16106.

Lemarié, S., Robert-Seilaniantz, A., Lariagon, C., Lemoine, J., Marnet, N., Jubault, M., Manzanares-Dauleux, M. J., and Gravot, A. 2015. Both the jasmonic acid and the salicylic acid pathways contribute to resistance to the biotrophic clubroot agent Plasmodiophora brassicae in Arabidopsis. Plant Cell Physiol. 56:2158-2168. 
Liu, P.-P., von Dahl, C. C., and Klessig, D. F. 2011. The extent to which methyl salicylate is required for signaling systemic acquired resistance is dependent on exposure to light after infection. Plant Physiol. 157: 2216-2226.

Liu, P.-P., Yang, Y., Pichersky, E., and Klessig, D. F. 2010. Altering expression of benzoic acid/salicylic acid carboxyl methyltransferase 1 compromises systemic acquired resistance and PAMP-triggered immunity in arabidopsis. Mol. Plant-Microbe Interact. 23:82-90.

Lovelock, D. A., Šola, I., Marschollek, S., Donald, C. E., Rusak, G., van Pée, K.-H., Ludwig-Müller, J., and Cahill, D. M. 2016. Analysis of salicylic acid-dependent pathways in Arabidopsis thaliana following infection with Plasmodiophora brassicae and the influence of salicylic acid on disease. Mol. Plant Pathol. 17:1237-1251.

Ludwig-Müller, J. 2014. Auxin homeostasis, signaling and interaction with other growth hormones during the clubroot disease of Brassicaceae. Plant Signal. Behav. 9:e28593.

Ludwig-Müller, J., Jülke, S., Geiß, K., Richter, F., Mithöfer, A., Šola, I., Rusak, G., Keenan, S., and Bulman, S. 2015. A novel methyltransferase from the intracellular pathogen Plasmodiophora brassicae methylates salicylic acid. Mol. Plant Pathol. 16:349-364.

Lulai, E. C., Suttle, J. C., Olson, L. L., Neubauer, J. D., Campbell, L. G., and Campbell, M. A. 2016. Wounding induces changes in cytokinin and auxin content in potato tuber, but does not induce formation of gibberellins. J. Plant Physiol. 191:22-28.

Lulsdorf, M. M., Yuan, H. Y., Slater, S. M. H., Vandenberg, A., Han, X., Zaharia, L. I., and Abrams, S. R. 2013. Endogenous hormone profiles during early seed development of $C$. arietinum and $C$. anatolicum. J. Plant Growth Regul. 71:191-198.

Manosalva, P. M., Park, S.-W., Forouhar, F., Tong, L., Fry, W. E., and Klessig, D. F. 2010. Methyl esterase 1 (StMES1) is required for systemic acquired resistance in potato. Mol. Plant-Microbe Interact. 23: 1151-1163.

Murmu, J., Wilton, M., Allard, G., Pandeya, R., Desveaux, D., Singh, J., and Subramaniam, R. 2014. Arabidopsis GOLDEN2-LIKE $(G L K)$ transcription factors activate jasmonic acid (JA)-dependent disease susceptibility to the biotrophic pathogen Hyaloperonospora arabidopsidis, as well as JA-independent plant immunity against the necrotrophic pathogen Botrytis cinerea. Mol. Plant Pathol. 15:174-184.

Oh, S.-K., Young, C., Lee, M., Oliva, R., Bozkurt, T. O., Cano, L. M., Win, J., Bos, J. I. B., Liu, H.-Y., van Damme, M., Morgan, W., Choi, D., Van der Vossen, E. A. G., Vleeshouwers, V. G. A. A., and Kamoun, S. 2009. In planta expression screens of Phytophthora infestans RXLR effectors reveal diverse phenotypes, including activation of the Solanum bulbocastanum disease resistance protein Rpi-blb2. Plant Cell 21: 2928-2947.

Park, S.-W., Kaimoyo, E., Kumar, D., Mosher, S., and Klessig, D. F. 2007. Methyl salicylate is a critical mobile signal for plant systemic acquired resistance. Science 318:113-116.
Rolfe, S. A., Strelkov, S. E., Links, M. G., Clarke, W. E., Robinson, S. J., Djavaheri, M., Malinowski, R., Haddadi, P., Kagale, S., Parkin, I. A. P., Taheri, A., and Borhan, M. H. 2016. The compact genome of the plant pathogen Plasmodiophora brassicae is adapted to intracellular interactions with host Brassica spp. BMC Genomics 17:272.

Sanchez-Vallet, A., Ramos, B., Bednarek, P., López, G., Piślewska-Bednarek, M., Schulze-Lefert, P., and Molina, A. 2010. Tryptophan-derived secondary metabolites in Arabidopsis thaliana confer non-host resistance to necrotrophic Plectosphaerella cucumerina fungi. Plant J. 63:115-127.

Shulaev, V., Silverman, P., and Raskin, I. 1997. Airborne signalling by methyl salicylate in plant pathogen resistance. Nature 385:718-721.

Strelkov, S. E., and Hwang, S.-F. 2014. Clubroot in the Canadian canola crop: 10 years into the outbreak. Can. J. Plant Pathol. 36 (sup1):27-36.

Vadassery, J., Reichelt, M., Hause, B., Gershenzon, J., Boland, W., and Mithöfer, A. 2012. CML42-mediated calcium signaling coordinates responses to Spodoptera herbivory and abiotic stresses in Arabidopsis. Plant Physiol. 159:1159-1175.

van Damme, M., Huibers, R. P., Elberse, J., and Van den Ackerveken, G. 2008. Arabidopsis DMR6 encodes a putative 2OG-Fe(II) oxygenase that is defense-associated but required for susceptibility to downy mildew. Plant J. 54:785-793.

van Hulten, M., Pelser, M., van Loon, L. C., Pieterse, C. M. J., and Ton, J. 2006. Costs and benefits of priming for defense in Arabidopsis. Proc. Natl. Acad. Sci. U.S.A. 103:5602-5607.

Van Wees, S. C. M., and Glazebrook, J. 2003. Loss of non-host resistance of Arabidopsis NahG to Pseudomonas syringae pv. phaseolicola is due to degradation products of salicylic acid. Plant J. 33:733-742.

Vlot, A. C., Dempsey, D. A., and Klessig, D. F. 2009. Salicylic acid, a multifaceted hormone to combat disease. Annu. Rev. Phytopathol. 47: 177-206.

Vlot, A. C., Liu, P. P., Cameron, R. K., Park, S.-W., Yang, Y., Kumar, D., Zhou, F., Padukkavidana, T., Gustafsson, C., Pichersky, E., and Klessig, D. F. 2008. Identification of likely orthologs of tobacco salicylic acidbinding protein 2 and their role in systemic acquired resistance in Arabidopsis thaliana. Plant J. 56:445-456.

Vos, I. A., Moritz, L., Pieterse, C. M. J., and Van Wees, S. C. M. 2015. Impact of hormonal crosstalk on plant resistance and fitness under multiattacker conditions. Front. Plant Sci. 6:639.

Whale, A. S., Huggett, J. F., Cowen, S., Speirs, V., Shaw, J., Ellison, S., Foy, C. A., and Scott, D. J. 2012. Comparison of microfluidic digital PCR and conventional quantitative PCR for measuring copy number variation. Nucleic Acids Res. 40:e82.

Widhalm, J. R., and Dudareva, N. 2015. A familiar ring to it: Biosynthesis of plant benzoic acids. Mol. Plant 8:83-97.

Win, J., Krasileva, K. V., Kamoun, S., Shirasu, K., Staskawicz, B. J., and Banfield, M. J. 2012. Sequence divergent RXLR effectors share a structural fold conserved across plant pathogenic oomycete species. PLoS Pathog. 8:e1002400. 